\title{
High Precision Quantum Control of Single Donor Spins in Silicon
}

\author{
Rajib Rahman, ${ }^{1, *}$ Cameron J. Wellard, ${ }^{2}$ Forrest R. Bradbury, ${ }^{3}$ Marta Prada, ${ }^{1}$ Jared H. Cole, ${ }^{2}$ \\ Gerhard Klimeck, ${ }^{1,4}$ and Lloyd C. L. Hollenberg ${ }^{2}$ \\ ${ }^{1}$ Network for Computational Nanotechnology, Purdue University, West Lafayette, Indiana 47907, USA \\ ${ }^{2}$ Center for Quantum Computer Technology, School of Physics, University of Melbourne, VIC 3010, Australia \\ ${ }^{3}$ Department of Electrical Engineering, Princeton University, Princeton, New Jersey 08544, USA \\ ${ }^{4}$ Jet Propulsion Laboratory, California Institute of Technology, Pasadena, California 91109, USA
}

(Received 1 March 2007; published 20 July 2007)

\begin{abstract}
The Stark shift of the hyperfine coupling constant is investigated for a P donor in Si far below the ionization regime in the presence of interfaces using tight-binding and band minima basis approaches and compared to the recent precision measurements. In contrast with previous effective mass-based results, the quadratic Stark coefficient obtained from both theories agrees closely with the experiments. It is also shown that there is a significant linear Stark effect for an impurity near the interface, whereas, far from the interface, the quadratic Stark effect dominates. This work represents the most sensitive and precise comparison between theory and experiment for single donor spin control. Such precise control of single donor spin states is required particularly in quantum computing applications of single donor electronics, which forms the driving motivation of this work.
\end{abstract}

DOI: 10.1103/PhysRevLett.99.036403

PACS numbers: 71.55.Cn, 03.67.Lx, 71.70.Ej, 85.35.Gv

Solid state quantum computer architectures have received considerable attention in recent years due to their promise of scalability and utilization of the vast knowledge and experience of the semiconductor fabrication industry. Several proposals employ the electronic states of phosphorus donors in silicon to encode qubits based on nuclear spin [1], electronic spin [2-4], or the electronic states of a singly ionized two donor system [5]. Two-dimensional architectures for such qubits have been proposed to demonstrate scalability strategies [6].

In Kane's original nuclear spin qubit proposal [1], and the more recent semiglobal control electron spin proposal [3], single-qubit operations are performed by electrically controlling the hyperfine interaction between the donor electron spin and the nuclear spin of the $\mathrm{P}$ impurity. To implement rigorous large-scale quantum algorithms protected by quantum error correction, the donor wave function-dependent hyperfine coupling constant needs to be controlled to a very high degree of precision. Recent experiments have made tremendous inroads towards achieving control of donors. In Ref. [7], charge relaxation times were measured in a two P donor device. In Ref. [8], coherent oscillations of $\mathrm{P}$ donor electron spins were measured by electrically detected magnetic resonance. Similar techniques were used in Ref. [9] to invesitgate the hyperfine interaction in the presence of lattice strain for Si grown on $\mathrm{Si}_{1-x} \mathrm{Ge}_{x}$. This work is motivated by a recent ESR experiment [10] that measured the Stark shifts for ${ }^{121} \mathrm{Sb}$ donors in silicon buried at a depth of $150 \mathrm{~nm}$ from an interface. Effective mass theory (EMT) inferred from $1 \mathrm{~s}$ manifold calculation of Ref. [11] predicts a quadratic Stark shift some 10 times greater than the measured result. This Letter investigates the Stark shift in the experimentally probed regime and shows that two sophisticated methods, namely, tight-binding (TB) and band minima basis (BMB), are able to predict the system control parameters very well. $\mathrm{The} \mathrm{Sb}$ and $\mathrm{P}$ donors in $\mathrm{Si}$ can be treated as analogous because their ground state binding energies differ only by $2.8 \mathrm{meV}$, and their $1 \mathrm{~s}$ manifold splittings due to valley-orbit coupling and local perturbations are also similar in nature [12]. This is important as both the TB and the BMB methods employed here are optimized for P donors. This agreement between theory and experiment for this measure of wave function control represents the most precise test of our understanding of donor systems and, in conjunction with the advancement in single atom fabrication technologies [13-16], represents an important step towards experimental realization of high precision control of donor spins in $\mathrm{Si}$.

Most of the approaches based on EMT have involved using hydrogenic envelope functions [17] with Bohr radii calculated analytically or fitted variationally. While Kettle et al. [18] used EMT to study the effects of electric fields and interfaces nonperturbatively with a Technology Computer Aided Design gate potential, this work ignored valley-orbit (VO) interaction responsible for lifting the degeneracies of the $1 s$ manifold. A number of authors $[11,19,20]$ used EMT to investigate the donor energy spectrum using symmetry arguments, perturbation theory, or the single or multivalley Schrödinger equation. In Ref. [11], Friesen considered valley-orbit coupling and field effects in the effective mass formalism, to solve a multivalley Schrödinger equation for the six $1 s$ states in the valley basis. Martins et al. [21] used a TB $s p^{3} s^{*}$ secondnearest-neighbor model to study effects of electric fields. In Ref. [22], the BMB approach was proposed and applied to the same problem. Calderon et al. [23] used EMT to investigate shuttling times of the donor electron between a 
donor-bound state and a surface bound state. Both TB and $\mathrm{BMB}$ approaches consider core effects and VO coupling explicitly to model impurities. The $\mathrm{TB}$ and the $\mathrm{BMB}$ methods are quite distinct in the sense that TB represents the Hamiltonian in real space, while BMB represents it in momentum space. The close agreement of the results from two such different methods of calculations, as shown in this work, emphasizes the fundamental nature of the impurity physics. The work presented here resolves significant limitations of the previous treatments by including realistically large device components with over $3 \times 10^{6}$ atoms in an atomistic treatment under realistic boundary conditions. This is also one of the first theoretical works on the Stark effect for P impurities to be benchmarked with a relevant experiment. While most theoretical approaches have concentrated on donor spectrum and wave functions near the ionization regime, little work has been done to explore the linear and quadratic Stark shifts of the hyperfine coupling far below the ionization regime for realistically large systems in the presence of interfaces.

The spin Hamiltonian of a donor electron in an applied electric field $\vec{\varepsilon}$ and a magnetic field $B_{0} \hat{z}$ is

$$
H_{z}=g(\vec{\varepsilon}) \mu_{B} B_{0} S_{z}+A(\vec{\varepsilon}) I_{z} S_{z},
$$

where $A(\vec{\varepsilon})$ and $g(\vec{\varepsilon})$ are the electric field-dependent hyperfine coupling and electron $g$ factor, respectively. An electric field distorts the shape of the donor wave function and modifies its angular momentum, which in turn modifies the $g$ factor. The field also pulls the donor wave function away from the impurity site, reducing $A(\vec{\varepsilon})$ which is proportional to $\left|\Psi\left(\vec{\varepsilon}, \vec{r}_{0}\right)\right|^{2}$, where $\vec{r}_{0}$ is the impurity site. The change in $A(\vec{\varepsilon})$ is parametrized as

$$
\Delta A(\vec{\varepsilon})=A(0)\left(\eta_{2} \varepsilon^{2}+\eta_{1} \varepsilon\right) .
$$

The quadratic Stark coefficient $\eta_{2}$ was measured to be $-3.7 \times 10^{-3} \mu \mathrm{m}^{2} / \mathrm{V}^{2}$ from the experiment by Bradbury et al. [10]. The EMT result inferred from Ref. [11] in Ref. [10] was $-2 \times 10^{-2} \mu \mathrm{m}^{2} / \mathrm{V}^{2}$. This value is used here for comparison, although we note that the result may change if $p$-type states are included in the EMT analysis. Since the spin-orbit Stark effect is small for practical magnetic fields, this Letter focuses on the behavior of $A(\vec{\varepsilon})$ only. An impurity placed far away from interfaces in an unstrained Si lattice experiences a dominant quadratic Stark effect for both $A(\vec{\varepsilon})$ and $g(\vec{\varepsilon})$. However, the presence of nearby interfaces or lattice strain can produce a non-negligible linear Stark effect $[24,25]$. The origin of the linear and quadratic Stark effects can be explained qualitatively from perturbation theory. The unperturbed ground state wave function for an impurity placed far away from interfaces has an approximate even symmetry. First order perturbation theory does not yield any energy correction if the perturbing Hamiltonian of a constant electric field is of odd symmetry. However, second order perturbation theory produces a quadratic dependence of the corrected energy on the field. If the impurity is very close to the interface, the even symmetry of the unperturbed wave function is broken. In such a case, first order perturbation theory also yields a linear dependence of the corrected energy on the electric field.

TB and BMB methods are separately used to calculate $A(\vec{\varepsilon})$. From each calculation, the ground state wave function $\Psi(\vec{\varepsilon}, \vec{r})$ is calculated in the presence of the field $\vec{\varepsilon}$. The hyperfine coupling $A(\vec{\varepsilon})$ is determined by

$$
\frac{A(\vec{\varepsilon})}{A(0)}=\frac{\left|\Psi\left(\vec{\varepsilon}, \vec{r}_{0}\right)\right|^{2}}{\left|\Psi\left(0, \vec{r}_{0}\right)\right|^{2}}
$$

In the range of low electric fields considered here, the excited states $(E$ and $T)$ are not expected to affect $A(\vec{\varepsilon})$ since this manifold is separated by $10 \mathrm{meV}$ from the ground $A_{1}$ state. While EMT-based approaches are concerned with contributions from valley-minima states only, TB and BMB approaches consider a more extensive Bloch structure of the material. TB and BMB also include VO interaction consistently, while most EMT treatments of VO are in violation of some of the assumptions made to derive the effective mass equation [22].

The BMB technique solves the Hamiltonian including an external electric field in a large but truncated basis of pure crystal Bloch states near the conduction band minima of a host obtained by the pseudopotential method. Since the discretized Schrödinger equation is solved in reciprocal space, the Fourier transform of the impurity potential is used. Near the impurity core, the potential is modified from its Coulombic nature to include central cell effects. The corrected potential produces a broader Fourier spectrum in $k$ space and couples different valleys to lift the $1 s$ degeneracies. The core potential in momentum space used in Ref. [22] is taken from Pantelides and Sah [26]. An overall quenching factor provides a one-parameter fit to the ground state energy from which the excited state energies and degeneracies compare well with experiment. After inclusion of the external field, the hyperfine coupling strength is obtained.

The standard technique in semiempirical TB [27] is to optimize a parameter set to accurately reproduce the bulk band structure of a host. Once this is done, the same set is used for atomistic modeling of any devices made of that host. For this work, the TB parameter set of $s p^{3} d^{5} s^{*}$ nearest-neighbor spin model for $\mathrm{Si}$ was optimized by a genetic algorithm [28]. The donor potential used is a screened Coulomb potential truncated to $U_{0}$ at the donor site. $U_{0}$ is treated as an adjustable parameter and found to be $4.33 \mathrm{eV}$ to obtain the experimental ground state energy of $45.6 \mathrm{meV}$ for Si:P. The potential due to the electric field is added to the diagonal of the Hamiltonian. The real space Hamiltonian with closed boundary conditions is solved by a parallel Lanczos algorithm in Nano Electronic Modeling Tool 3D [29] for the donor wave function. 

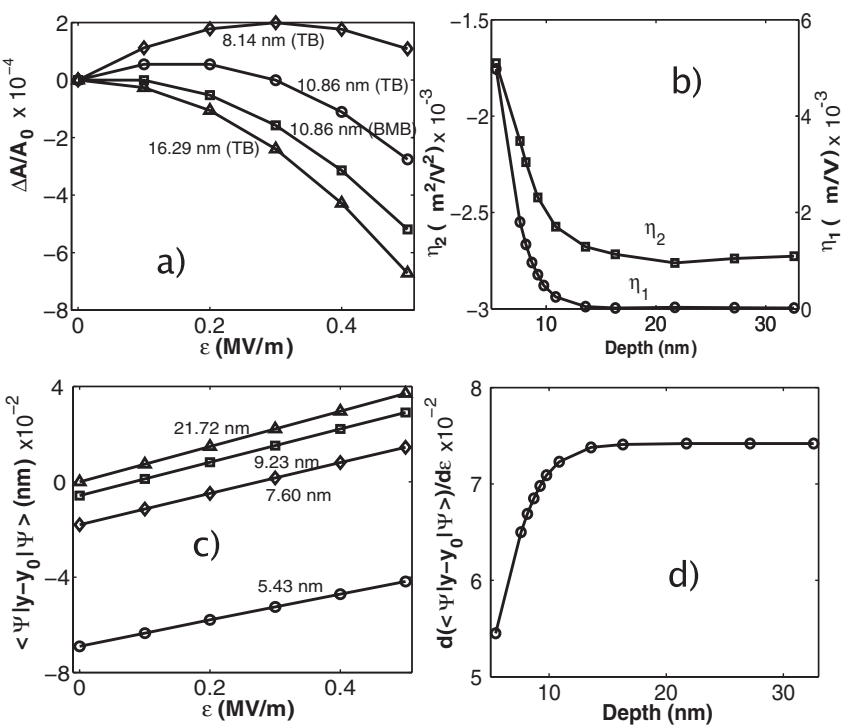

FIG. 1. (a) Electric field response of hyperfine coupling at various impurity depths (BMB and TB). (b) Quadratic (lefthand axis) and linear (right-hand axis) Stark coefficients with depth (TB). (c) Mean position of the ground state electron distribution (dipole moment) as a function of the electric field (TB). (d) The electric field gradient of the dipole moments [i.e., the slopes of the lines in (c)] with respect to depth (TB).

Both methods described here are applicable to other hosts and impurities. To describe other group V impurities, the hydrogenic Coulomb potential is still valid in the bulk, but the core corrections need to be adjusted to reflect correct experimental binding energies.

Figure 1 summarizes the effects of the electric field and the interface on the donor electron. The TB calculations use a domain of $32 \mathrm{~nm} \times 65 \mathrm{~nm} \times 32 \mathrm{~nm}$ zinc blende lattice with $3.45 \times 10^{6}$ atoms. The distance between the impurity and the interface is varied parallel to the electric field. The BMB calculation assumes a maximal depth of $10.86 \mathrm{~nm}$ from the interface and employs a basis set of 7986 states. The choice of this depth is dictated by the ease of computation as it is sufficiently deep to nullify surface effects while not too deep to make the problem computationally intractable. In $\mathrm{TB}$, a range of depths from 5 to $32 \mathrm{~nm}$ have been considered. For each TB data point, the typical computation times require about 7 hours on 20 CPUs [30]. Figure 1(a) shows the variation of $\Delta A(\vec{\varepsilon})$ with electric field for various impurity depths. The data are fitted to the quadratic equation of (2). As the depth increases, the quadratic coefficient $\eta_{2}$ approaches a constant value, while the linear coefficient $\eta_{1}$ becomes negligible [Fig. 1(b)]. For small impurity depths, $\eta_{1}$ is comparable to $\eta_{2}$, which results in a shift of the peak of the parabola in Fig. 1(a) towards a nonzero electric field. If the linear Stark effect is negligible, an applied electric field has two effects on the ground state wave function: (i) a decrease in the peak amplitude of the wave function at the impurity site, reflected by a decrease in $A(\vec{\varepsilon})$ in Fig. 1(a) for higher depths, and (ii) a shift in the mean position of the wave function opposite the electric field, giving rise to a nonzero dipole moment as shown in Fig. 1(c). The dipole moments vary linearly with the electric field, and their slopes approach a constant value as the depth increases [Fig. 1(d)]. The wave function plots of Fig. 2 also demonstrate these effects. The larger distortions of the wave function at higher electric fields explain the increasing dipole moments.

If the impurity is close to the interface, the wave function is distorted even at zero electric field. This distortion comes from changes in the slope of the part of the wave function between the impurity and the interface. Also the mean position of the wave function shifts farther as the distance between the impurity and the interface is reduced. These effects give rise to an asymmetric charge distribution about the impurity, and a nonzero dipole moment is observed even at $\varepsilon=0$. This is verified by the shift of the $y$ intercept of the lines in Fig. 1(c) for small impurity depths. In effect, the interface behaves like an electric field pushing the donor electron away from it. The strength of this field increases as the impurity is placed closer to the interface. This means that a higher electric field directed away from the interface is needed to counteract these interface effects and to restore the decreasing behavior of $A(\vec{\varepsilon})$, as demonstrated in Fig. 1(a).

Some of the numerical results can be explained qualitatively with the perturbation model using a basis of unperturbed impurity wave functions $\left\{\Psi_{m}^{0}\right\}$, where $m$ indexes increasing binding energy ( $m=0$ being the ground state). For an electric field given by $q \varepsilon y$, the wave function corrected to first order is expressed as

$$
\left|\Psi_{0}^{1}\right\rangle=\left|\Psi_{0}^{0}\right\rangle-q \varepsilon \sum_{m \neq 0} \sum_{i} \frac{\left\langle\Psi_{m, i}^{0}|y| \Psi_{0}^{0}\right\rangle}{E_{0}^{0}-E_{m, i}^{0}}\left|\Psi_{m, i}^{0}\right\rangle,
$$

where $i$ is the degeneracy index for a state $m$. The dipole moment $D=q\left\langle\Psi_{0}^{1}|y| \Psi_{0}^{1}\right\rangle$ is then given by

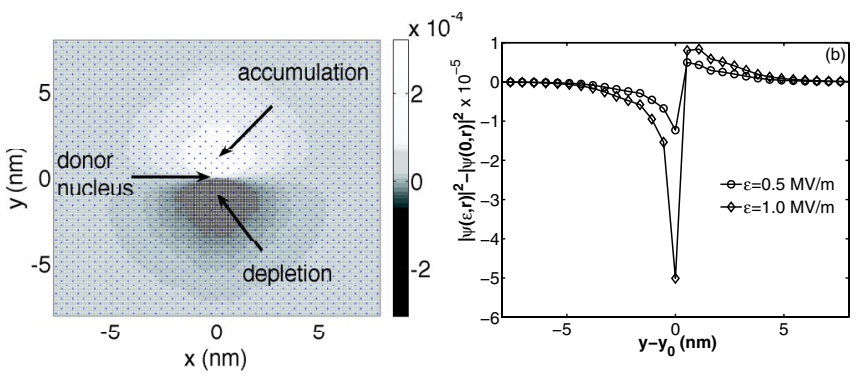

FIG. 2 (color online). (a) Electric field-induced differential map of donor electron wave probability density $\left|\Psi\left(\varepsilon, z_{0}\right)\right|^{2}-$ $\left|\Psi\left(0, z_{0}\right)\right|^{2}$ shown as a $2 \mathrm{D}$ cut through the impurity center at $z_{0}=$ $16.29 \mathrm{~nm}$ for $\varepsilon=0.5 \mathrm{MV} / \mathrm{m}$. The electric field is directed along the negative $y$ axis. (b) $1 \mathrm{D}$ cut though the center of the impurity parallel to the electric field showing the differential map of the probability density for two different electric fields. 
TABLE I. Comparison of the quadratic Stark coefficients from experiment, EMT, BMB, and TB.

\begin{tabular}{lcr}
\hline \hline \multicolumn{1}{c}{ Method } & Depth $(\mathrm{nm})$ & $\eta_{2}\left(\mu \mathrm{m}^{2} / \mathrm{V}^{2}\right)$ \\
\hline Experiment (Sb) [10] & 150 & $-3.7 \times 10^{-3}$ \\
EMT (P) [11] & $\infty$ & $-2 \times 10^{-2}$ \\
BMB (P) & 10.86 & $-2.74 \times 10^{-3}$ \\
TB (P) & 10.86 & $-2.57 \times 10^{-3}$ \\
& 21.72 & $-2.76 \times 10^{-3}$ \\
\hline \hline
\end{tabular}

$$
\begin{aligned}
D= & q\left\langle\Psi_{0}^{0}|y| \Psi_{0}^{0}\right\rangle-2 q^{2} \varepsilon \sum_{m \neq 0} \sum_{i} \frac{\left|\left\langle\Psi_{m, i}^{0}|y| \Psi_{0}^{0}\right\rangle\right|^{2}}{E_{0}^{0}-E_{m, i}^{0}} \\
& +q^{2} \varepsilon^{2} \sum_{m_{1,2} \neq 0} \sum_{i, j} \frac{\left\langle\Psi_{0}^{0}|y| \Psi_{m_{1}, i}^{0}\right\rangle\left\langle\Psi_{m_{2}, j}^{0}|y| \Psi_{0}^{0}\right\rangle}{\left(E_{0}^{0}-E_{m_{1}, i}^{0}\right)\left(E_{0}^{0}-E_{m_{2}, j}^{0}\right)}
\end{aligned}
$$

The quadratic term in $\varepsilon$ can be neglected compared to the other two terms for small $\varepsilon$. This results in a linear function with intercept and slope depending on $\left\langle\Psi_{0}^{0}|y| \Psi_{0}^{0}\right\rangle$ and $\left|\left\langle\Psi_{0}^{0}|y| \Psi_{m, i}^{0}\right\rangle\right|^{2}\left(E_{0}^{0}-E_{m, i}^{0}\right)^{-1}$, respectively. For an impurity far away from the interface, the unperturbed ground state wave function is of even symmetry, the expression for the intercept evaluates to zero, and the expression for the slope assumes a constant value. On the other hand, for small impurity depths, the unperturbed wave function is distorted by the proximity to the interface. Since the ground state has no longer an even symmetry, the intercept evaluates to a nonzero quantity, in consistence with Fig. 1(c). The transition probabilities between the ground state and the excited states are also changed due to these distortions, causing the slope of the dipole moment to vary with depth, in consistence with Fig. 1(d). The same reasoning explains why the quadratic Stark coefficient approaches a constant value and the linear Stark coefficient becomes negligible with increasing depth.

Table I gives the comparison between theory and experiment and shows that the EMT estimate of $\eta_{2}$ differs by an order of magnitude, while TB and BMB estimates agree closely with each other and the experiment. The BMB estimate for a depth of $10.86 \mathrm{~nm}$ is already close to the converged TB estimate, although the peak $\Delta A(\vec{\varepsilon})$ at a nonzero $\varepsilon$ in Fig. 1(a) indicates that the BMB estimate would improve even more at greater depths.

In summary, the Stark shift of the hyperfine coupling for $\mathrm{Si}: \mathrm{P}$ in the presence of interfaces is analyzed from two very different theories (BMB and TB). The results of both methods are consistent and agree well with the experimental data for $\mathrm{Si}: \mathrm{Sb}$, thereby providing the most sensitive test of our understanding of shallow donor quantum control to date. For future work, both TB and BMB can be optimized for Sb donors, and the Stark shifts can be investigated in the presence of interfaces as well as lattice strain and a gate structure more closely resembling that of the experiment. In conclusion, we emphasize the need for very close interaction between theory and experiment to accomplish the high precision control required in quantum computing.

This work was supported by the Australian Research Council, the Australian Government, and the U.S. National Security Agency (NSA), Advanced Research and Development Activity (ARDA), and the Army Research Office (ARO) under Contract No. W911NF-04-1-0290. Part of this work was done at JPL, Caltech under a contract with NASA. NCN/nanohub.org computational resources were used.

*rrahman@purdue.edu

[1] B. E. Kane, Nature (London) 393, 133 (1998).

[2] R. Vrijen et al., Phys. Rev. A 62, 012306 (2000).

[3] C. D. Hill et al., Phys. Rev. B 72, 045350 (2005).

[4] R. deSousa et al., Phys. Rev. A 70, 052304 (2004).

[5] L. C. L. Hollenberg et al., Phys. Rev. B 69, 113301 (2004).

[6] L. C. L. Hollenberg et al., Phys. Rev. B 74, 045311 (2006).

[7] S. E. S. Andresen et al., Nano Lett. 7, 2000 (2007).

[8] A. R. Stegner et al., Nature Phys. 2, 835 (2006).

[9] H. Huebl et al., Phys. Rev. Lett. 97, 166402 (2006).

[10] F. R. Bradbury et al., Phys. Rev. Lett. 97, 176404 (2006).

[11] M. Friesen, Phys. Rev. Lett. 94, 186403 (2005).

[12] A. K. Ramdas et al., Rep. Prog. Phys. 44, 1297 (1981).

[13] J. R. Tucker et al., Solid State Electron. 42, 1061 (1998).

[14] S. R. Schofield et al., Phys. Rev. Lett. 91, 136104 (2003).

[15] F. J. Ruess et al., Nano Lett. 4, 1969 (2004).

[16] D. N. Jamieson et al., Appl. Phys. Lett. 86, 202101 (2005).

[17] W. Kohn and J. M. Luttinger, Phys. Rev. 98, 915 (1955).

[18] L. M. Kettle et al., Phys. Rev. B 68, 075317 (2003).

[19] A. Debernardi et al., Phys. Rev. B 74, 035202 (2006).

[20] G. D. J. Smit et al., Phys. Rev. B 68, 193302 (2003).

[21] A. S. Martins et al., Phys. Rev. B 69, 085320 (2004).

[22] C. J. Wellard and L.C. L. Hollenberg, Phys. Rev. B 72, 085202 (2005).

[23] M. J. Calderon et al., Phys. Rev. Lett. 96, 096802 (2006).

[24] G. M. Guichar et al., Phys. Rev. B 5, 422 (1972).

[25] D. K. Wilson and G. Feher, Phys. Rev. 124, 1068 (1961).

[26] S. T. Pantelides and C. T. Sah, Phys. Rev. B 10, 621 (1974).

[27] J. C. Slater and G. F. Koster, Phys. Rev. 94, 1498 (1954).

[28] T. B. Boykin et al., Phys. Rev. B 69, 115201 (2004).

[29] G. Klimeck et al., Comput. Model. Eng. Sci. 3, 601 (2002).

[30] nanoHUB.org computational resource of a 256-node 3.3 GHz Pentium Irvindale PC cluster was used. 